\title{
The Influence of Natural Environment on the Forms of Traditional Dwellings-the Case of Qinghai-Tibet Plateau
}

\author{
Yanke Li ${ }^{1}$, Siyi Lin ${ }^{1}$, Shiru Liu ${ }^{1}$, Sihua Deng ${ }^{1}$, Yi Huang ${ }^{2, ~ *, ~ J i n ~ T a o ~}{ }^{2, ~ * ~}$ \\ ${ }^{1}$ Department of Architecture, South China University of Technology, Guangzhou, China \\ ${ }^{2}$ State Key Lab of Subtropical Building Science, Department of Architecture, South China University of Technology, Guangzhou, China
}

Email address:

335120873@qq.com (Yi Huang), arjtao@scut.edu.cn (Jin Tao)

${ }^{*}$ Corresponding author

\section{To cite this article:}

Yanke Li, Siyi Lin, Shiru Liu, Sihua Deng, Yi Huang, Jin Tao. The Influence of Natural Environment on the Forms of Traditional Dwellings - the Case of Qinghai-Tibet Plateau. International Journal of Architecture, Arts and Applications. Vol. 6, No. 3, 2020 , pp. 47-55. doi: $10.11648 /$ j.ijaaa.20200603.13

Received: April 29, 2020; Accepted: June 10, 2020; Published: August 13, 2020

\begin{abstract}
Known as "the third pole of the world", Qinghai-Tibet Plateau occupies an important position among the global plateaus. Due to the complex landform and climate, the types of the traditional dwellings in Qinghai-Tibet Plateau are diverse. This paper classifies the traditional dwellings of Qinghai-Tibet plateau based on their architectural plane, structure, material, function, etc. Then remote sensing images combined with field survey are adopted to spatially divide various types of traditional dwellings, and draw their spatial division map. On this basis, the spatial distributions of various dwelling types are fused with the natural environment information of the Qinghai-Tibet Plateau, and the patterns and laws of the distribution of various types of dwellings are analyzed. Finally, through the analysis of relevant impact factors, the causes of the temporal and spatial distribution of dwelling forms are revealed. The results show that the natural environment has a decisive influence on the form of the traditional dwellings through geographical features, temperature, precipitation and solar radiation. The overlap of the spatial distribution of various types of dwellings, and the generation of the architectural form such as sloping blockhouse and "Zhuangke" dwelling are the effective responses to the special natural and cultural environment of Qinghai-Tibet Plateau.
\end{abstract}

Keywords: Qinghai-Tibet Plateau, Natural Environment, Dwelling Types, Spatial Distribution

\section{Introduction}

Known as "the ridge of the world," Qinghai-Tibet plateau is the highest one of the three natural staircases of China, with an average elevation of more than 4,000 meters [1]. Restricted by the natural environment, different areas of Qinghai-Tibet plateau have formed different dwelling types, which have their own characteristics. At present, studies on the influence of natural environment to the architectural forms of dwellings in Qinghai-Tibet plateau are not abundant. Zongwei $\mathrm{Xu}$ made a detailed discussion on the functional classification of ancient Tibetan buildings from three aspects of historical causes, site layout and architectural characteristics, and systematically summarized the development of ancient buildings in various regions of Tibet, the characteristics of various buildings and construction skills [2]. Minan Yang explored the morphological characteristics and ecological strategies of "Zhuangke" dwellings in Hehuang area of Qinghai province from three aspects of the structural characteristics of the building, the layout of the courtyard, the materials and craftsmanship [3]. Wenhe Cui gave an all-round and multi-angle in-depth explanation of Qinghai rural dwellings, summed up the architectural characteristics of the rural dwellings in the four natural comprehensive areas of eastern Qinghai Province, Huanhu, Haixi and Hainan. From a cultural perspective, he made an in-depth and systematic summary of the architectural characteristics of the 6 resident ethnic groups of Han, Tibet, Hui, Tu, Sara, and Mongolia from a cultural perspective [4]. Benteng Liu made a in-depth analysis and comparison of the differences and characteristics of the spatial structure and spatial form of traditional villages and dwellings in Longzhong, Longdongnan, Gannan, and Hexi areas of Gansu from the perspectives of nature, society, and humanities [5]. Yunnan Provincial Design Institute's "Yunnan Dwellings" 
writing team introduced and sorted out the types of dwellings in Yunnan [6]. Yin Liang discusses the uniqueness and diversity of the architectural landscape of the Tibetan, Qiang, $\mathrm{Yi}$, Bai, and Dai people in the southwest of china from a cultural overview [7].

Generally speaking, most of the existing studies on dwelling types of Qinghai-Tibet plateau mainly concentrated on history, society and national culture. Researches involving the impact of natural environment on the form of buildings are mainly case analysis. In this paper, by adopting the research methods of remote sensing image analysis, field investigation, literature analysis etc., the dwelling types of the Qinghai-Tibet plateau are classified, the spatial distribution of them and the geographical and climatic conditions of Qinghai-Tibet Plateau are put together, by which we try to explore the relationship between the natural environment and the distribution of the dwelling forms of Qinghai-Tibet plateau.

\section{The traditional Dwelling Types in Qinghai-Tibet Plateau}

\subsection{Flat Roof Blockhouse}

Flat roof blockhouse is a typical dwelling type in Qinghai-Tibet Plateau, which are built with materials such as soil, wood, stone, etc $[8,9]$. They are built along the mountain, with ladder-shaped appearance and rectangular flats, and have gorgeous decoration and rich colors. Generally, the dwelling are 2-3 floors, and each floor is about $2.7 \mathrm{~m}$, they are closed, masonry and have no wooden columns between the walls. The windows of the dwellings are generally opened inward, characterized by few and small, staggered positions. The dwellings are stone-wood structure, which features are "no wood outside, no soil inside". Generally, the ground floor is for raising livestock and storing, the second floor is for living, and the number of rooms in the dwellings are calculated by the columns. Functionally, the best location indoors is to arrange a Buddhist temple, followed by bedrooms and a kitchen, besides, there is a drying platform outside where is a place of daily life, and is also used for threshing and drying food when necessary.

\subsection{Sloping Blockhouse}

Generally, sloping blockhouse are independent or detached, with a gentle double slope roof and their flats are square or rectangle [10]. Sloping blockhouse are mostly 3 stories, with a low ground floor for livestock, the second floor is used for living, which is centered on the fire pond, and the furnitures are arranged around the fire pond. The space under the pitched roof of the third floor is used for storing firewood, fodder and sundries. In terms of materials, the external walls can be made of crushed stone, rubble, pebbles, rammed earth, wooden boards, bamboo hedges, wicker hedges, etc [6]. Sloping blockhouse are Stone-wood structure system, with the timber beams and rafters of the pitched roofs rest on the gable, and their roofs are usually made of wood or slate. Besides, their appearances are sedate and gorgeous, with abundant decorations on their wooden components such as doors and windows.

\section{3. "Zhuangke" Dwelling}

"Zhuangke" dwelling is a traditional and common dwelling form in agricultural areas of eastern Qinghai, with flat roofs smoothed with grass-mud, which construction materials are earth, stone, wood, etc $[11,12]$. Their exterior walls are thick and heavy, and their construction methods are simple and economical. During construction, the "Zhuangke" walls encircling the entire courtyard are rammed first, and then set the wooden structure against the walls to build the houses, and the rear partition walls of the houses are usually set at a distance from the "Zhuangke" walls so as to form an air gap between the two walls, which is not only convenient for construction, but also plays a crucial role in thermal insulation. The dwellings are mostly south or east, and their layout has three forms: quadrangle courtyard, triples and double-sided houses. Among the buildings in the courtyard, the hall is the main building. In front of the hall, there is a large outdoor space under the eaves for family rest, children's play, etc. The two sides of the hall are built into wing rooms, with eaves porches in front of them. And there is a reversely-set house in front of the hall, which is often used for livestock or as a warehouse.

\subsection{Courtyard Dwelling}

The courtyard dwelling is formed under the influence of the Han-style "Siheyuan" dwelling culture, and there are two types of courtyard dwelling in Qinghai-Tibet Plateau. The first type has a spacious courtyard, which surrounded by relatively independent buildings in four directions. They are mostly brick-wood structures, and their roofs are made of wood beams, purlins and rafters [13, 14]. Larger dwellings have gorgeous decoration such as brick carving, wood carving, calligraphy and painting [15]. The other is smaller "Tianjing" courtyard, which is composed of several small patios. These dwellings are mostly two-story, 7.5 meters high, with adobe walls and tiled roofs, and their cornices and ridges are the key decorative parts.

\section{5. "Chuandou" Wooden Dwelling}

"Chuandou" wooden dwelling is one of the structural forms of Chinese traditional wooden dwelling, which is characterized by a clear division of load-bearing and maintenance structure. When building a house, first set a wooden frame, then fill the walls [16]. They have two forms of facade, one is a earthen wall and the other is a wooden board. Their roofs are mostly "Xuanshan" style, which are thinner than those of northern dwellings. The structures of "Chuandou" wooden dwelling are flexible, which can be elevated and cantilevered, and have a strong adaptability to the terrain and environment. The overhead "Chuandou" wooden dwelling are also called "Ganlan" houses, which appearance are rough and simple in nature, when viewed from a distance, we can only the see the low bamboo mat walls and the hanging 
"Xuasnhan" roof are erected on densely arranged columns.

\section{6. "Jinggan" Wooden Dwelling}

The appearance of these dwellings is simple and rugged, with fewer windows open on their facade. It is constructed by stacking layers of wood whose cross-sections are round, square, or hexagonal. During construction, the timbers cross each other at the corners of the walls, roughly protruding the stacked logs, with no paint on the surfaces, and the contact surfaces of the wood are usually partially chopped for stability and water resistance [17-19]. In some cold areas, mud is applied to the wooden seams to prevent wind and cold. Nails are rarely used; their components are mostly connected by tenon-and-mortise.
Generally, their roofs have a gentle slope, and their flats are

\subsection{Plateau Cave Dwelling}

The caves in Qinghai-Tibet Plateau make full use of the terrain. They usually choose high cliff faces of different platforms to dig, and then make it flat in front of the door to form small platforms or roads. Usually, several or dozens of caves are arranged form a row on the same cliff surface in a ladder shape. The orientations of the doors vary with the turning and bending of the cliff faces. The flats of the cave have different shapes such as square, rectangle and circle. There are soil stoves in the rooms, soots on the inner walls, and smoke holes through the doors for some caves.

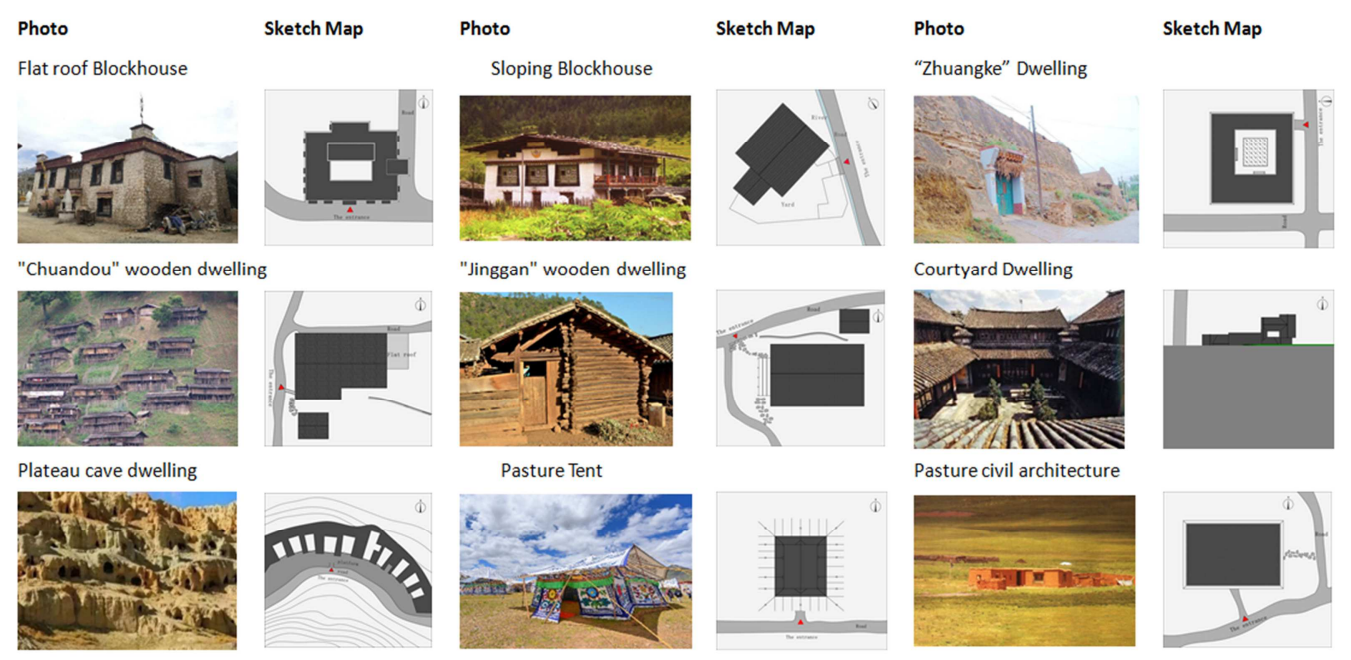

Figure 1. The types of vernacular dwellings in Qinghai-Tibet Plateau mainly rectangular in shape.

\subsection{Pasture Tent}

Pasture tent is a main dwelling type of herdsmen in Qinghai-Tibet Plateau, which includes tent, Kazak yurt and Mongolian yurt. The characteristics of the dwellings are light and easy to disassemble, and they are well adapted to the lifestyle of the local herders. Tents are the most common way of living for herders, and they can be divided into winter tent and summer tent according to the climate change of four seasons. The flats of tents are mostly rectangular, with simple furnishings inside. The clear height of the tent is only about 1.6-1.9 $\mathrm{m}$, and there is a skylight on the top of the tent for ventilation. The structural of the tent is herringbone-shaped slope on both sides, which is fixed by ropes tied wooden piles, its features are keep the wind and rain from breaking. The Mongolian yurt is a tap-wood structure dwelling which flat is a circle with a diameter of $4 \mathrm{~m}$. The central clear height of the yurt is about 2.2 meters, and there is a skylight on the top of the yurt for lighting. During construction, build a grid-like enclosed space with the poles cross in both directions first, then cover them with white felt, canvas outside, finally tied the straps. Kazak yurt are similar to Mongolian yurt in structure and flat, while the difference is that the Kazak yurt are dome shaped and held up by poles, while the Mongolian yurt are Supported by straight poles and their roofs are tapered.

\subsection{Pasture Civil Architecture}

Pasture civil architecture are simple structured dwellings for herders to settle in winter pastures in Qinghai-Tibet Plateau [2]. The scales of these dwellings are not large, and most of them have no courtyard. Due to the shortage of building materials in pastoral areas, most of them are built with loess adobe, a small part of them are constructed of stone and wood materials. In some areas, even the turf is cut into square barriers and used as a building material for walls of the dwellings. The doors and windows of the dwellings are small, and the windows are trumpet-shaped trapezoid, which are convenient for observing livestock. For there are no carving on the exterior walls and interior wood components, the appearance of the vernacular dwellings are rough and simple in nature.

\section{Distribution of Dwelling Types in Qinghai-Tibet Plateau}

\subsection{Flat Roof Blockhouse}

Flat roof blockhouse is the largest spatial covered and most numerous type of dwelling in Qinghai-Tibet Plateau. It covers the most areas of Tibet, western and southern Qinghai, 
southern Gansu, western Sichuan, and northern Yunnan, with a total of 131 counties (districts), 20 cities (autonomous states) in 5 provinces (autonomous regions). In terms of the types of economic production, flat roof blockhouse are intensively distributed in the agricultural counties, semi-agricultural and semi-livestock counties, partially distributed in the towns of livestock counties, and a small dispersion in other areas.

\subsection{Sloping Blockhouse}

Sloping blockhouse are mainly distributed in the southeast of Tibet, Zhongdian in northern Yunnan, southern Gansu, Ganzi and Aba in western Sichuan, with a total of 27counties (districts), 10 cities (autonomous states) in 4 provinces (autonomous regions). In terms of the economic background, sloping blockhouse are mainly locate in semi-agricultural and semi-livestock counties, with a small amount in some livestock counties. The distribution of sloping blockhouses are not extensive, and they are coincident with the flat-top blockhouse, "Chuandou" wooden dwelling and "Jinggan" wooden dwelling in different degrees, meanwhile their morphological characteristics also combine some features of them, so it can be considered as a transitional dwelling type in Qinghai-Tibet Plateau.

\section{3. "Zhuangke" Dwelling}

"Zhuangke" dwelling are mainly distributed in the eastern Qinghai, the northern and the western Gansu, with a total of 26 counties (districts), 10 cities (autonomous states), 2 provinces (autonomous regions). Geographically, their distribution ranges are not extensive but relatively concentrated, and have certain overlaps with the blockhouse and courtyard dwelling. Moreover, their forms and layout draw on some characteristics of the blockhouse's and the courtyard dwelling's respectively, therefore it can be considered as another kind of transitional building type in Qinghai-Tibet Plateau.

\subsection{Courtyard Dwelling}

According to statistics, the courtyard dwelling are mainly distributed in parts of eastern Qinghai, western and northern Gansu, northern Yunnan and western Sichuan, with a total number of 69 counties (districts), 25 cities (autonomous states), 4 provinces (autonomous regions). Geographically, courtyard dwelling are widely and consecutively distributed. Besides, they have different degrees of spatial overlaps with the traditional wooden structure houses, and "Zhuangke" dwelling, so it can be considered as a type of marginal dwelling in Qinghai-Tibet Plateau.

\section{5. "Chuandou" Wooden Dwelling}

"Chuandou" wooden dwelling are mainly distributed in the southeast of Tibet, southern Gansu, western Sichuan and the northwest of Yunnan, with a total number of 36 counties (districts), 13 cities (autonomous states), 3 provinces (autonomous regions). The distribution of "Chuandou" wooden dwelling in Qinghai-Tibet Plateau are not extensive but relatively concentrated, and there are certain spatial overlaps with various wooden structure dwellings, which also indicate to some extent that "Chuandou" wooden dwelling is another kind of marginal architecture in Qinghai-Tibet Plateau.

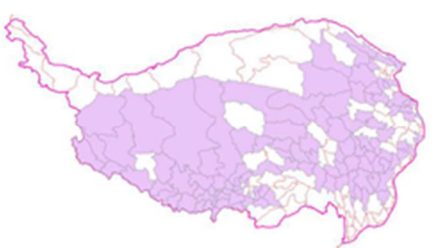

Flat roof Blockhouse
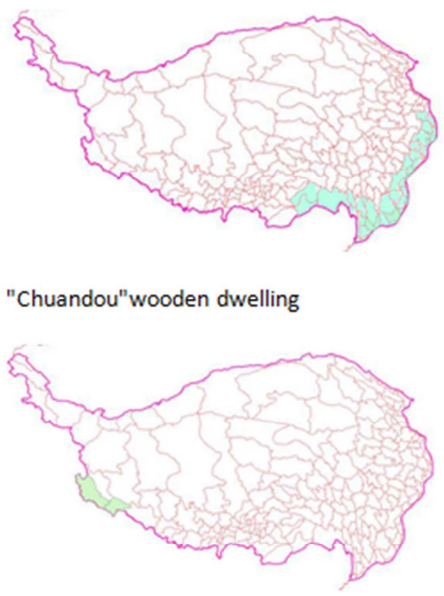

Plateau cave dwelling

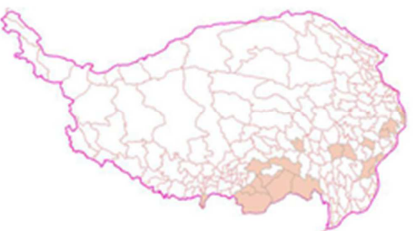

Sloping Blockhouse
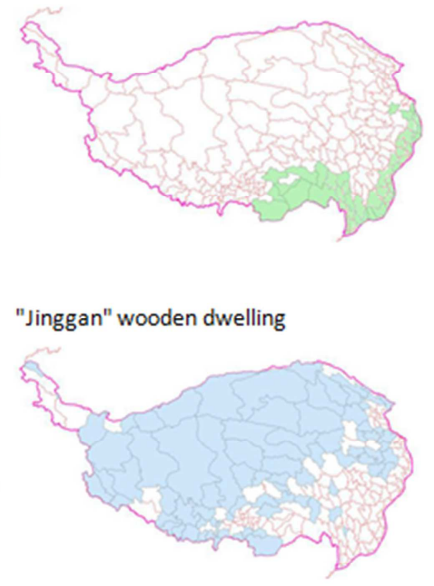

Pasture Tent
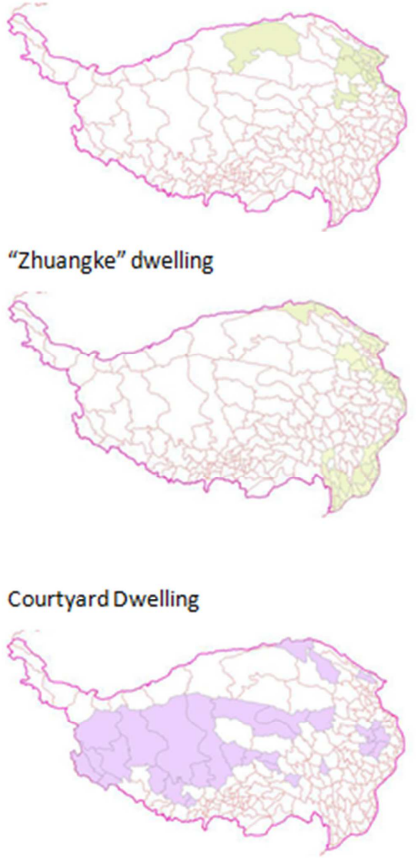

Pasture civil architecture

Figure 2. The spatial distribution of various types of vernacular dwellings in Qinghai-Tibet Plateau. 


\section{6. "Jinggan" Wooden Dwelling}

"Jinggan" wooden dwelling are mainly distributed in the southeast of Tibet, southern Gansu, western Sichuan, and the northwest of Yunnan, with a total number of 45 counties (districts), 17 cities (autonomous states), 4 provinces (autonomous regions). The spatial distribution of "Jinggan" wooden dwelling is relatively concentrated but not extensive, and they have a high degree of coincidence with the "Chuandou" wooden dwelling and courtyard dwelling. This also explains indirectly that the "Jinggan" wooden dwelling belong to the marginal dwelling of the Qinghai-Tibet Plateau as well.

\subsection{Plateau Cave Dwelling}

Plateau caves are only distributed in the two counties of Purang and Zada in the Ali region of Tibet [2]. There are also some cave dwellings scattered in the Hengduan Mountain region of eastern Tibet, which serve as temporary dwellings for herdsmen. The distribution of plateau cave dwelling in Qinghai-Tibet Plateau is extremely single, which can be considered as a special type of dwelling in Qinghai-Tibet Plateau.

\subsection{Pasture Tent}

Pasture tent are mainly concentrated in northern and southern Tibet, most areas in Qinghai, northwest Sichuan, southeast Gansu, and part areas of Xinjiang, a total of 132 Counties (districts), 26 cities (autonomous states), 6 provinces (autonomous regions). From an economic perspective, pasture tent are concentrated in animal husbandry counties in various provinces, and there are also many distributions in semi-agricultural and semi-livestock husbandry counties. The pasture is another main type of houses in Qinghai-Tibet Plateau.

\subsection{Pasture Civil Architecture}

This type of dwelling is mainly distributed in the northern Tibet, the west and south of Qinghai, western Sichuan, the southeast of Gansu, and southern Xinjiang, a total of 57 counties (districts), 18 cities (autonomous states), 5 provinces (autonomous regions). The spatial coverage of civil architecture in pastoral areas are relatively wide, and they have a high degree of overlap with the spatial distribution of tents, which can be considered as a kind of transitional architecture type between the flat roof blockhouse and the pasture tent.

\section{Impact of Natural Environment on the Morphology of Dwellings in Qinghai-Tibet Plateau}

Judging from the superposition of the spatial distribution of various dwellings and natural environment information, we can find that the spatial forms of these dwellings are closely related to the natural environment characteristics of the Qinghai-Tibet Plateau. The natural environment plays a vital role in shaping the residences in Qinghai-Tibet Plateau.

(1) The blockhouse has a large natural geographical span and is the main dwelling type of the Qinghai-Tibet Plateau. They are distributed from extreme arid, semi-arid and natural arid areas to humid, humid -semi-humid natural areas [8]. However, blockhouses in different areas have significant differences in architectural forms. Statistics show that flat roof blockhouse are mainly distributed in arid and semi-arid areas with an annual rainfall of 75 600 mm, an annual average temperature of $-5.6 \sim 0^{\circ} \mathrm{C}$, and solar radiation of $6200 \sim 8000 \mathrm{MJ} / \mathrm{M}^{2}$. While the sloping blockhouse are mainly distributed in humid, humid-semi-humid regions with annual rainfall of $600 \sim 4000 \mathrm{~mm}$, annual average temperature of $0 \sim 14^{\circ} \mathrm{C}$, and solar radiation of $3800 \sim 7000 \mathrm{MJ} / \mathrm{M}^{2}$. In addition, in the transitional areas of arid and humid climate with annual rainfall of $600-1000 \mathrm{~mm}$, annual average temperature of $0 \sim 14^{\circ} \mathrm{C}$, and solar radiation of $3800 \sim 6200 \mathrm{MJ} / \mathrm{M}^{2}$, co-existence of flat roof blockhouse and sloping blockhouse will occur in some areas. This indicates to some extent that the morphology of the blockhouses are affected by climate, especially the rainfall; the form of the blockhouses are different in flat top and the slope top with the annual rainfall of $600 \mathrm{~mm}$ as the dividing line.

(2) The geographical distribution of the pastoral dwellings also have a large span in arid, semi-arid, humid, humid-semi-humid natural areas, and they have a high adaptability to the climatic environment and a wide range of adaptation to rainfall, temperature, and solar radiation. They are distributed in areas with annual precipitation of $25 \sim 3000 \mathrm{~mm}$, annual average temperature of $-5.6 \sim 12^{\circ} \mathrm{C}$, and total solar radiation of $5200 \sim 8000 \mathrm{MJ} / \mathrm{M}^{2}$. On the other hand, the civil architecture in the pastoral area can be explained to be a variant of the pasture tent to adapt to the alpine weather in winter, which is an indirect reflection of the influence of temperature on the morphological characteristics of the dwellings. The different morphological characteristics of pastoral civil architecture in different natural areas indirectly reflect the impact of regional precipitation and temperature conditions on the morphological characteristics of dwellings: pasture civil architecture with annual precipitation of $25 \sim 400 \mathrm{~mm}$, annual average temperature of $-5.6 \sim 2^{\circ} \mathrm{C}$, and total solar radiation of $66008000 \mathrm{MJ} / \mathrm{M}^{2}$ have flat roofs with soil and turf as the outer protection; in humid and semi-humid pasture areas with annual rainfall of 500 to $800 \mathrm{~mm}$, annual average temperature of 0 to $12^{\circ} \mathrm{C}$, and total solar radiation of 5200 to $6000 \mathrm{MJ} / \mathrm{M}^{2}$, the water and heat conditions are good, thus the outer protection of civil buildings are made of wood with a slope top which keep the building warm in winter and cool in summer. 
(3) The traditional wooden dwellings are not widely distributed and belong to the marginal building type. They are mainly concentrated in the humid, humid-semi-humid natural areas in the southeast of Qinghai-Tibet Plateau. The annual rainfall here is more than $600 \mathrm{~mm}$; the average annual temperature is $2 \sim 18^{\circ} \mathrm{C}$, and the solar radiation is $3800 \sim 6800 \mathrm{MJ} / \mathrm{M}^{2}$. Rich rainfall and good light and heat conditions make the forest resources developed here, which provide good environmental conditions for the survival and development of traditional wooden dwelling. It is notable that in the humid, humid-semi-humid agricultural-pastoral-forestry interlaced area, there is a certain overlap in the spatial distribution of the sloping blockhouse and traditional wooden dwelling. This is the result of the dual effects of the natural environment and the cultural environment. The rainy environment determines the common feature of the slope roof, while the different nationalities and religious practices determine the choice of different dwelling types. The interaction between the two promotes the integration of flat roof blockhouse and traditional wooden dwelling.

(4) The courtyard dwellings also belong to the marginal building types of the Qinghai-Tibet Plateau. These dwellings are distributed in semi-arid areas and humid, humid-sub-humid areas. It is notable that the morphological characteristics of courtyard dwelling in different climates show certain differences. In the semi-arid area with an annual rainfall of 300 to $400 \mathrm{~mm}$, an annual average temperature of 2 to $8^{\circ} \mathrm{C}$, and solar radiation of 5400 to $6600 \mathrm{MJ} / \mathrm{M}^{2}$, the brick and soil resources are sufficient, and the climate is characterized by dry, cold and windy weather, low rainfall, strong radiation. While in humid, humid-sub-humid areas with annual rainfall of $500 \sim 1000 \mathrm{~mm}$, annual average temperature of $6 \sim 12^{\circ} \mathrm{C}$, and solar radiation of $3800 \sim 5400 \mathrm{MJ} / \mathrm{M}^{2}$, the forest resources are abundant, and the climate is characterized by heavy rainfall and damp weather. Therefore, the courtyard dwellings in arid areas are made of brick or civil structures. The appearance features are thick and heavy with wide courtyards for daylighting. The dwellings in the humid and semi-humid area are mostly made by timber frame with earthen walls and courtyards; they are lightweight and convenient for sun-shade and ventilation. The above objectively proves that the morphological characteristics of residential dwellings are the direct manifestation of temperature, solar radiation and rainfall climatic conditions.

(5) The courtyard dwellings also belong to the marginal building types of the Qinghai-Tibet Plateau. These dwellings are distributed in semi-arid areas and humid, humid-sub-humid areas. It is notable that the morphological characteristics of courtyard dwelling in different climates show certain differences. In the semi-arid area with an annual rainfall of 300 to $400 \mathrm{~mm}$, an annual average temperature of 2 to $8^{\circ} \mathrm{C}$, and solar radiation of 5400 to $6600 \mathrm{MJ} / \mathrm{M}^{2}$, the brick and soil resources are sufficient, and the climate is characterized by dry, cold and windy weather, low rainfall, strong radiation. While in humid, humid-sub-humid areas with annual rainfall of $500 \sim 1000 \mathrm{~mm}$, annual average temperature of $6 \sim 12^{\circ} \mathrm{C}$, and solar radiation of $3800 \sim 5400 \mathrm{MJ} / \mathrm{M}^{2}$, the forest resources are abundant, and the climate is characterized by heavy rainfall and damp weather. Therefore, the courtyard dwellings in arid areas are made of brick or civil structures. The appearance features are thick and heavy with wide courtyards for daylighting. The dwellings in the humid and semi-humid area are mostly made by timber frame with earthen walls and courtyards; they are lightweight and convenient for sun-shade and ventilation. The above objectively proves that the morphological characteristics of residential dwellings are the direct manifestation of temperature, solar radiation and rainfall climatic conditions.

(6) "Zhuangke" dwelling are only distributed in natural areas with annual rainfall of $25 \sim 600 \mathrm{~mm}$, annual average temperature of $2 \sim 8^{\circ} \mathrm{C}$, and solar radiation of $5400 \sim 7000 \mathrm{MJ} / \mathrm{M}^{2}$. The climate in this area is characterized by hot summers, cold winters, dry, windy, and sandy weather. Different from the cold climate in the hinterland of the Qinghai-Tibet Plateau and the warm climate in the southeast of the Qinghai-Tibet Plateau, it belongs to a transitional intermediate climate zone. Therefore, Zhuangke's morphological characteristics are similar to that of the blockhouse' $\mathrm{s}$ with a closed appearance, heavy stability, and squareness, which are easy to protect against wind and coldness, and they have the introverted characteristics of a courtyard-style house which are closed outside and open inside.

(7) Plateau cave dwelling are only distributed in the two counties of Purang and Zada in the Ali region of Tibet. The annual rainfall here is only $75 \sim 200 \mathrm{~mm}$; the average annual temperature is $0 \sim 2^{\circ} \mathrm{C}$, and the total solar radiation is as high as $7400 \sim 8000 \mathrm{MJ} / \mathrm{M}^{2}$. The specific geographical environment, temperature, rainfall, and solar radiation make this area arid, alpine, lack of vegetation resources, and extremely developed in soil forests, which also create specific conditions for the emergence of the plateau cave dwellings. However, Plateau cave dwellings are formed under special conditions in few places, and cannot represent a universal regional feature, so they are only a special dwelling type in the specific environment of the partial local area of the Qinghai-Tibet Plateau. 


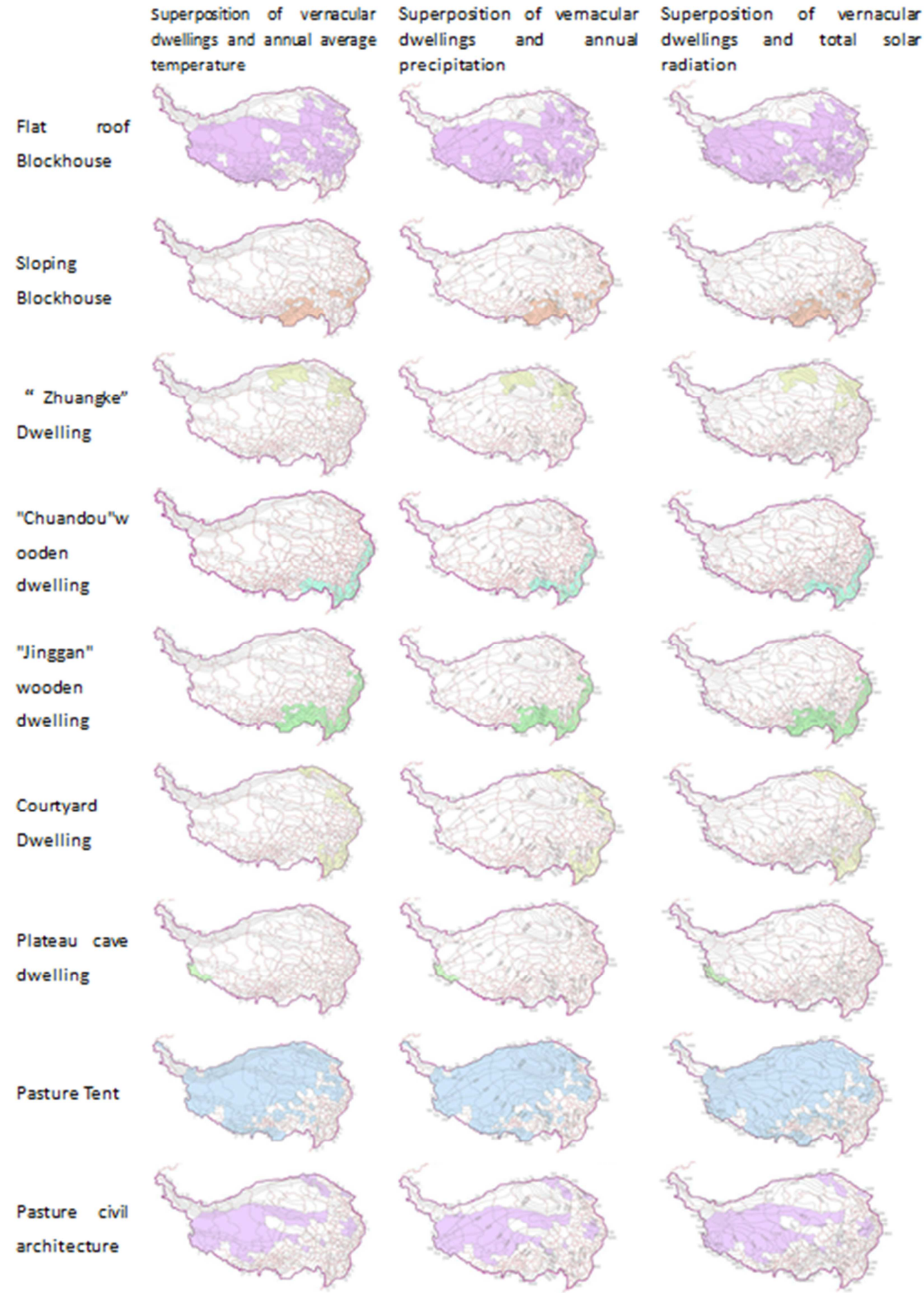

Figure 3. The spatial distribution of various types of vernacular dwellings overlay with meteorological map.

\section{Conclusion and Discussion}

The distribution pattern of the Qinghai-Tibet Plateau dwelling forms is formed under the local special geographical environment and climatic conditions. This paper classifies the vernacular dwellings of the Qinghai-Tibet Plateau and draws the spatial distribution map of various dwelling types, which has been fused with the natural environment information of the Qinghai-Tibet Plateau to analyze how the natural environment affects the distribution pattern of the dwelling forms through the geographical features, temperature, precipitation, and solar radiation, etc. The main conclusions are as follows:

(1) Geographical environment is the dominant factor that influences the distribution of dwelling types: the 
different geographical environments in different regions lead to different objects and methods of engaging in the material production, in turn, it determines the structural form and functional structure of vernacular dwellings, which has been reflected in the different distributions of residential dwelling types. In the pastoral areas, pasture tent and civil architecture which adapt to the traditional and modern grazing forms have been created; different forms of blockhouses have been created in the semi-agricultural and semi-pastoral areas, and various traditional wooden dwellings and courtyard dwellings have emerged in the forest area as well. There are 108 livestock and semi-livestock counties among the 234 counties in the Qinghai-Tibet Plateau, which fundamentally determines that the blockhouses and the pastoral dwellings are the main building types of the Qinghai-Tibet Plateau; the phenomenon that the forest coverage rate of $5 \%$ of the total area of the Qinghai-Tibet Plateau and its marginal position, together with the fact that the cultivated land is less than $0.22 \%$ of the total area in the Qinghai-Tibet Plateau, determines that the traditional wooden dwelling, courtyard dwelling, and "Zhuangke" dwelling are the marginal building types in the Qinghai-Tibet Plateau. As for the special local natural environment of Purang County and Zada County, it determines that the plateau cave is a special dwelling type in the Qinghai-Tibet Plateau area.

(2) Precipitation, temperature, and solar radiation shape the morphological characteristics of the dwellings. From the southeast to the northwest of the Qinghai-Tibet Plateau, the temperature and rainfall drop gradually with the solar radiation increasing gradually, and the morphological characteristics of dwellings changes correspondingly: first of all, from the traditional wooden dwelling to the blockhouse dwelling, the height gets lower and lower; the space division and the window size also gets smaller and smaller; the wall turns thicker and thicker, and the appearance of the dwellings are getting closed gradually. To the north of Tibet, the low yurts become the main dwellings, and the plateau cave dwelling even appear. Courtyard dwelling have also changed from the elegant and light houses in Yunnan to the closed, thick and spacious houses in Xiahe, Gansu. Secondly, the roofs of the houses have changed from the light and thin slope roof of the traditional wooden structure houses and the courtyard dwellings to the slope top of the traditional wooden houses, the intersection of the sloping blockhouse and the flat blockhouse, and then completely changed to the blockhouses with closed ribbed grass-mud flat tops. Finally, the choices of the dwelling materials are also quite different; they change from wood to raw soil materials such as earth and stone.

(3) It is the dual response to the special natural and cultural environment of the Qinghai-Tibet Plateau that leads to the emergence of Sloping blockhouse which is the integration of traditional wooden dwelling and blockhouses located in Nyingchi, Mêdog County, Tibet and Zhongdian, Dêqên County, Yunnan, and the type of Zhuangke dwellings which combined courtyard dwellings with blockhouses in Qinghai and Gansu. Meanwhile, this is also the main reason for the overlapping distribution of courtyard dwellings, traditional wooden dwelling, flat roof blockhouse and sloping blockhouse in northern Yunnan and western Sichuan.

\section{Acknowledgements}

This work was supported by the National Key R\&D Programme of China (Grant Number: 2017YFC0702402).

\section{References}

[1] Zheng, Zhao. (2017). Characteristics of the natural environment of the Qinghai-Tibet Plateau, (06): 13-22.

[2] Xu.(2016). Ancient Tibetan architecture. Beijing: China Building Industry Press, (01): 445.

[3] Yang, Cao, Cui. (2019). Ecological strategy of "ZhuangKe" dwellings in Hehuang area, Urban and Rural Construction, (22): 64-65.

[4] Cui. (2018). Research on the Design Method of Qinghai Rural Residential Renewal Suitability. Shanghai: Tongji University Press, (06): 287.

[5] Liu, Zhang, Li. (2018). Traditional villages in Gansu. Nanjing: Southeast University Press, (11): 345.

[6] Yunnan Design Institute "Yunnan dwellings" writing group. (2018). Yunnan dwellings. Beijing: China Building Industry Press, (01): 352.

[7] Liang. (2018). Research on the architectural landscape of ethnic minorities in Southwest China. Beijing: Atomic Energy Press Beijing Book Publishing Department, (08): 195.

[8] Cheng, Zhou, Dong. (2020). The regional expression of Maoshi epidermis in Tibetan Qiang blockhouse. Urban Architecture, 17 (2): 60-62.

[9] Zhang, Zhang. (2019). Investigation and research of blockhouse building. Henan Building Materials, (5): 334-335.

[10] Zhang, Li. (2017). Research on Tibetan dwellings culture - Based on the investigation of folk customs of four economic forms in Tibe. Qinghai Nationalities Studies, 0 (2).

[11] Fang, Wang, Jin. (2017) The ecological architecture experience and green renewal design of the traditional "Zhuangke" dwellings in Hehuang, Qinghai. Huazhong Architecture, 35 (8): 49-52.

[12] Wang, Lei. (2020) Research on Green Strategy of Traditional Villages in Hehuang Region Based on Climate Analysis. Journal of Western Human Settlement Environment, 35 (2): 104-110.

[13] Pang, Zhang, Zhang. (2020). Comparison of typical traditional dwellings courtyard culture in Gansu Province under the space syntax. Resource Development and Market, 36 (2): 174-179. 
[14] Pang, Zhang, Zhang. (2019). Research on the regional differentiation of the single flat structure of traditional dwellings in Gansu. Regional research and development 38 (6): 158-164.

[15] Gong, Li. (2018) A Comparative Study on the Characteristics of Gansu Traditional dwellings_—Building a New Mode of Modern "Gansu Characteristic Residential Buildings". Residential Technology, 38 (6): 11-14.

[16] Li, Wang, Dai. (2019) Mechanical performance analysis of single-layer "Chuandou" structure dwellings in Yunnan. Jiangxi Building Materials, (12): 73-74, 76.
[17] Shi Tuo. (2019). A preliminary study on traditional dwellings building technology in Yunxian, Yunnan. Ancient building garden technology, (4): 10-12.

[18] Li, Wang, Dai. (2018). Basic structure and seismic performance analysis of two-story wooden dwellings in Yunnan. Low temperature building technology, 40 (9): 52-56.

[19] Wei, Yu. (2019). Interpretation and inheritance of construction methods of Bai people's dwellings in Yunnan. Art Science and Technology, 2 (8): 60-61. 